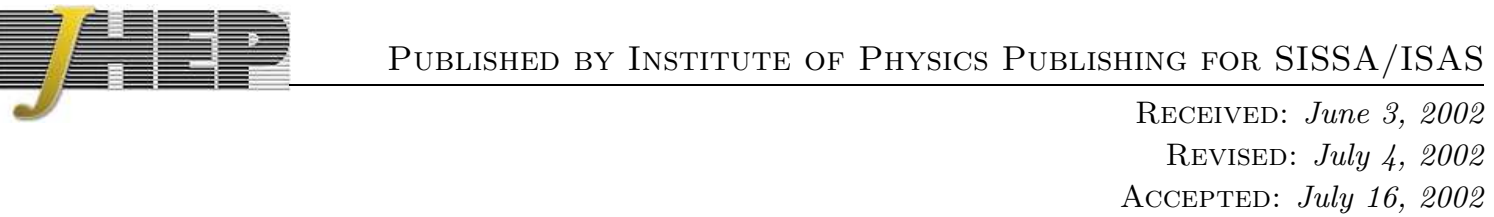

\title{
Reality, measurement and locality in quantum field theory
}

\author{
Daniele Tommasini \\ Departamento de Física Aplicada, Área de Física Teórica, Universidad de Vigo \\ 32004 Ourense, Spain \\ E-mail: daniele@uvigo.es
}

\begin{abstract}
It is currently believed that the local causality of Quantum Field Theory (QFT) is destroyed by the measurement process. This belief is also based on the Einstein-PodolskyRosen (EPR) paradox and on the so-called Bell's theorem, that are thought to prove the existence of a mysterious, instantaneous action between distant measurements. However, I have shown recently that the EPR argument is removed, in an interpretation-independent way, by taking into account the fact that the Standard Model of Particle Physics prevents the production of entangled states with a definite number of particles. This result is used here to argue in favor of a statistical interpretation of QFT and to show that it allows for a full reconciliation with locality and causality. Within such an interpretation, as Ballentine and Jarret pointed out long ago, Bell's theorem does not demonstrate any nonlocality.
\end{abstract}

Keywords: Space-Time Symmetries, Gauge Symmetry, Models of Quantum Gravity, Standard Mode]. 


\section{Contents}

1. Introduction 1

2. Reality and completeness: the EPR paradox 2

3. The supposed proofs of nonlocality 3

4. Photons uncertainty, reality and the EPR paradox

5. The interpretation of QFT 5

6. The EPR correlations in QFT 9

7. Conclusions 12

\section{Introduction}

Quantum Field Theory (QFT) QFT has been argued to be the unique reasonable realization of a Relativistic Quantum Mechanics [1], and it can be thought to be the "low energy" limit of any possible Theory of Everything. In fact, an extremely successful description of Particle Physics, valid at least up to energies $\sim 100 \mathrm{GeV}$, has been obtained with the Weinberg - Salam Standard Model [2], which is based on the hypothesis that all the known interactions (except gravity) can be derived from a local symmetry $\mathrm{SU}(3) \times \mathrm{SU}(2) \times \mathrm{SU}(1)$. The field equations define a perfectly Lorentz-invariant, causal and local theory [1, 2]. Of course, these characteristics are most welcome in a relativistic world. However, they are

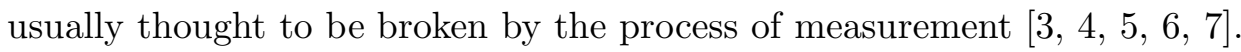

In fact, the measurement process is usually described by a "collapse" of the state vector of the system, which after measurement is projected onto an "out" state, an eigenstate of the observed quantities (e.g. the momenta and helicities of the observed particles). This collapse postulate works to obtain correct theoretical predictions, however it implies that the measurement process itself is a sort of discontinuous process that cannot be described with the smooth, linear evolution that applies to all known interactions (this is the socalled "measurement problem", see refs. 8, 6] for reviews showing different points of view). Moreover, the "collapse" is described as a global effect, involving at the same time all the space.

Is such a supposed nonlocality a real physical property of any quantum theory, and possibly of Nature (as it is usually supposed today)? Or is that merely a problem of the global collapse description, i.e. of the "interpretation" of the theory? Is it possible to avoid the collapse and the measurement problem, and describe the measurement process with the same local QFT laws that describe particle interactions? 


\section{Reality and completeness: the EPR paradox}

In their famous 1935 paper [9], EPR provided two important concepts that have greatly influenced the subsequent research in the foundations of the quantum theory.

I. A "condition of completeness" for any acceptable theory: "every element of the physical reality must have a counterpart in the physical theory" [9].

II. A definition of the objective "physical reality": "If, without in any way disturbing a system we can predict with certainty (i.e. with probability equal to unity) the value of a physical quantity, then there is an element of physical reality corresponding to this physical quantity" [9].

These concepts were aimed at dealing with the main difficulty of Quantum Mechanics (QM): the particles and the physical quantities are usually not definite (i.e. they have no "reality") until a measurement is performed. ${ }^{1}$ However, EPR pointed out that even ordinary QM allows for some elements of physical reality: if a system is prepared in an eigenstate of a given observable, we can predict with certainty that the result of the measurement of that observable will be the corresponding eigenvalue: there is then an element of objective physical reality corresponding to that observable.

In Classical Physics, all the physical quantities have a definite value in a given system at a given time (although in the case of macroscopic systems we usually do not know such a value and can only use a statistical description). In the usual "orthodox" interpretation of QM, however, it is supposed that the state vector completely describes the state of a system, and this does not allow for a certain prediction of the results of the measurements of two noncommuting observables, such as the position and the momentum. Given a state, unavoidably there are some observables (heuristically, a "half" of the set of the observables) that do not have a reality, and their measurement in an ensemble of copies of the system prepared in this state will show a nonvanishing dispersion. Are these nondiagonalized physical quantities really undefined on the single copy of the system that is under consideration? Or is this uncertainty merely a consequence of an unavoidable lack of knowledge, as in the classical, statistical description of the macroscopic systems? In the latter case, the QM description should be completed by introducing some new, "hidden" variables, possibly allowing for an underlying determinism.

The brilliant argument developed by EPR was aimed at resolving this dilemma. In fact, they invented a thought-experiment for which QM itself predicted that two incompatible observables (position and momentum in their original formulation; two noncommuting components of the spin in a version due to Bohm [10]) were given a simultaneous reality. They argued that this result was in contradiction with the assumption that the wave function completely described the physical reality.

Although all the present discussion can be easily generalized to EPR experiments involving any kind of particles, hereafter I will only consider the so-called "EPR-Bohm" thought-experiment [10, 11]. Two spin 1/2 particles, A and B, are created in coincidence

\footnotetext{
${ }^{1}$ This is just a generalization of the so-called wave-particle duality.
} 
in a spin-singlet state, and are detected by the detectors $O_{A}$ and $O_{B}$ in opposite directions. The measurement of a given component $\vec{S} \cdot \vec{a}$ of the spin of particle A (or of B) along a unit vector $\vec{a}$ can give the values $\pm \hbar / 2$, each with probability $1 / 2$. However, if $\vec{S} \cdot \vec{a}$ is measured on $\mathrm{A}$ and found, say, equal to $+\hbar / 2$, not only does this give a physical reality to such a spin component on $\mathrm{A}$; in fact, momentum conservation allows for predicting with certainty the value $-\hbar / 2$ for the same spin component on B. EPR assumed that the physical reality on $B$ is independent of what is done with $A$, which is spatially separated from the former (this assumption has been called Einstein's Locality). Since a certain prediction for the considered spin component on B was allowed without in any way disturbing particle $B$, therefore the spin component $\vec{S} \cdot \vec{a}$ has a reality on $B$. By repeating this argument for any component of the spin, we deduce that all the spin components $\left(S_{x}, S_{y}, S_{z}\right)$ have a simultaneous physical reality on particle B. But this contradicts ordinary QM as based on the wave function, where only one component of the spin of a given particle can be definite.

This result is a rigorous consequence of two assumptions, as Einstein himself noticed in 1949 [12]:

"The paradox forces us to relinquish one of the following two assertions:"

1. the description by means of the wave function is complete,

2. the physical realities of spatially separated objects are independent of each other.

The incompatibility of statements 1) and 2) has also been called EPR theorem (see e.g. refs. [8, 13, 6]).

Since "Einstein's locality" assertion 2) was considered unquestionable by EPR, they deduced that the wave function did not provide a complete description of the state of a system. This was a very powerful argument in favor of deterministic (and local) hidden variable theories. In such theories, all the observables have a definite value in the single system that is under consideration. The dispersion of the probability distributions observed in the repetition of the experiment on an ensemble of identically prepared systems is merely a "statistical mechanics" effect. Such a "cryptodeterminism" (see page 155 of ref. [13]) would explain the fact that the measurement of a component of the spin of A apparently has a deterministic effect on the distant measurement of the same component of the spin of B: both results would actually be the deterministic consequence of the common production of the two particles.

\section{The supposed proofs of nonlocality}

In the mid sixties, Bell proved that deterministic hidden variable theories were actually viable, but they could not reproduce all the results of QM [14], unless they implied an instantaneous action at a distance [15]. He proposed a set of "Bell's inequalities" for the spin correlations in a realization of the EPR-Bohm experiment, that were violated by QM and respected by any local deterministic hidden variable theory. Since the actual experiments [16] confirmed the predictions of QM, local determinism was ruled out. This result will be called hereafter the "original Bell's theorem". 
Therefore, it was deduced that QM was a complete theory, and EPR Theorem forced to conclude that it had to be a "nonlocal" theory. ${ }^{2}$ Hereafter, I will call this argument the "EPR+Bell" proof of nonlocality, since it is based on both EPR and Bell's theorems. ${ }^{3}$

Moreover, in the last several years there has been a proliferation of "generalized Bell's Theorems", claiming that the observed violation of Bell's inequalities was sufficient in itself to prove the existence of an instantaneous influence between distant measurements.

As a result of these two proofs, there has been increasing agreement within quantum physics experts ${ }^{4}$ on the conclusion that Nature is EPR paradoxical. "Quantum nonlocality" is considered an experimental evidence, and it is really believed that the two distant measurements in the EPR experiments do actually influence each other instantaneously. The "speed of quantum information" has even been "measured" to be greater than $1.5 \times 10^{4} c$ [7].

Einstein could never accept the existence of such a "spooky action at a distance", which is incompatible with special relativity. In fact, this supposed "quantum nonlocality" is the main origin of the widespread belief that the Quantum Theory is incompatible with

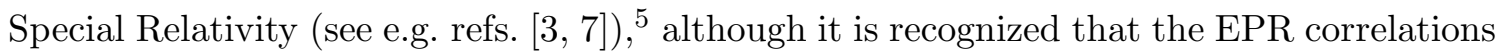
do not allow for superluminal signaling, e.g. they cannot be used to synchronize clocks (see ref. [6] for a review).

In fact, any kind of "nonlocality" or instantaneous "distant influence" is unacceptable in a relativistic world: due to the relativity of the simultaneity, suitable observers would describe this influence (whatever it is) as an effect of the future on the past [18]. Fortunately for science, there is a way to completely reconcile the quantum theory with locality, causality and special relativity. This solution is quite natural and is based merely on known physics.

\section{Photons uncertainty, reality and the EPR paradox}

The EPR argument, as described above, and (as far as I know) all the subsequent treatments of the EPR paradox, have assumed that it was actually possible to prepare a system of two entangled particles. In fact, for the EPR argument it is crucial that the measurement on A implies a certain prediction for B without disturbing B: thus different noncommuting observables on $\mathrm{B}$ are forced to have a physical reality. ${ }^{6}$

\footnotetext{
${ }^{2}$ This conclusion also seemed to be suggested by the very fact that it was actually possible to reproduce the QM results using an explicitly nonlocal deterministic theory that was found by Bohm [17].

${ }^{3}$ Many of the existing supposed proofs of nonlocality are reelaborations or variations of this argument.

${ }^{4}$ There have been remarkable exceptions, however, mainly represented by researchers resisting giving up local hidden variable theories. All of them would deserve a citation, but I can only give space to the argument published in 1987 by Ballentine and Jarret [18], whose main conclusions are still valid today, although they are almost universally ignored.

${ }^{5}$ As Ballentine and Jarrett remarked, "such a contradiction, if indeed one exists, between two fundamental and exceedingly well verified theories would constitute a major crisis in theoretical physics. One may wonder why so few physicists are apparently concerned (or even aware of) such a crisis" [18]. Surprisingly, the same consideration can be repeated today, to an even greater extent.

${ }^{6} \mathrm{EPR}$ explicitly asked for a unit probability for this prediction, but we can replace their requirement with the weaker one: with probability $1-\epsilon$, where $\epsilon$ may be made arbitrarily small [8]. The following discussion remains true.
} 
However, I have recently proved that this assumption is not correct [11]. In fact, the Standard Model of Particle Physics predicts that it is not possible to produce a state having a definite particle content: given the process that produces $A$ and $B$ alone, QFT theory predicts a nonvanishing and finite probability for the creation of $A$ and $B$ plus additional photons 11]. In other words, given the initial system that is used for the production of A and $\mathrm{B}$, the rate for the processes involving additional photons is a given finite number that cannot be made arbitrarily small.

As a consequence, I argued that it is never possible to make a certain prediction of the spin state of B by merely measuring a spin component of particle A in an EPR experiment. Energy, momentum and angular momentum conservation do not hold for the (sub)system made of the two particles $\mathrm{A}$ and $\mathrm{B}$, since additional photons can carry such conserved quantities. By detecting $\mathrm{A}$, it is not even possible to predict if particle $\mathrm{B}$ will actually be caught in the opposite direction [11].

The conclusion may seem surprising: QFT satisfies the EPR criterion of completeness without the need for hidden variables and without necessarily violating Einstein's condition of locality for the physical reality [11]. In other words, the EPR argument is removed because of the fact that QFT allows for less EPR reality than was believed. This solution, based on the greater uncertainty of QFT, is completely opposite to the local determinism solution based on hidden variables. Incidentally, since it is impossible to prepare a state with a definite number of photons, and since such an uncertainty for any given process cannot be made arbitrarily small, we may even argue that it is not possible to give a physical reality (not even locally) to any observable, except to the charges and masses (that are the invariants of the theory). ${ }^{7}$

Therefore, we no longer have to decide between statements 1) and 2) in EPR theorem: QFT remains an EPR-complete theory and yet it does not satisfy condition 1), that implicitly assumed the entangled two particle state vector. Therefore QFT can satisfy 2) in $\mathrm{EPR}$ theorem. In particular, the EPR+Bell proof of nonlocality is removed.

This result is particularly significant since it relies on only well-established physics and it does not depend on any particular interpretation of the theory. However, it is clear that a decision about the locality of QFT can be made only after examining the description of the measurement process. If (and only if) this can be done in a local way, without postulating any global collapse of the state vector, then QFT will be automatically a causal and local theory. ${ }^{8}$ We will now look for an interpretation that executes this program.

\section{The interpretation of QFT}

According to the previous discussion, QFT can be considered an EPR-complete theory. If

\footnotetext{
${ }^{7}$ In QFT, the usual assumption that the state of a system can be prepared by measuring a Complete Set of Commuting Observables is also incorrect, e.g. it is impossible to determine the occupation numbers of the photon states arising from a given process. Of course, the usual Fock space basis of QFT, diagonalizing all the occupation numbers, can still be used as a mathematical tool.

${ }^{8}$ Incidentally, it is obvious that the orthodox interpretation will be nonlocal, since it postulates such a global collapse. I do not need Bell's Theorems to believe that.
} 
we do not introduce any hidden variables or new physics beyond QFT, we are left with two possibilities for an interpretation:

I. The state vector applies to the single system. Now, since the state vector of a free system evolves deterministically in Quantum Mechanics and in QFT, the joint state of the measuring apparatus (including all the "environment" which interacts with it) and the object system after the measurement has to be determined by that before the measurement. In particular, the position of the pointer, i.e. the result of the measurement, has to be the deterministic consequence of the initial conditions, and the only possible source of indeterminism is the unavoidable statistical ignorance of the state of the environmental variables. Since the QFT laws are local, the world would be intrinsically deterministic and local. This possibility is logically consistent, but it seems to be unable to provide a satisfactory solution to the "measurement problem" (see below).

The "orthodox" interpretations [6, 13] introduce the collapse postulate in order to reconcile the assumption that the state vector applies to the single system with the fact that the measurement gives sharp results. The measurement process is then considered a magical process, different from the physical interactions that are well described by QFT. I think that this ad hoc assumption is very unnatural in QFT; it also contradicts the experience in particle physics detectors, that shows that the measurement process is determined by the same strong and electroweak interactions that are described by QFT. Moreover, the collapse of the state vector has to simultaneously involve all the space and explicitly violates locality and Lorentz symmetry. This is unacceptable in a relativistic world, as discussed above. Paradoxically, in spite of all these contradictions and absurdities, orthodox interpretations are currently the most common choice. Up to now, this may have been justified precisely by the pressure of both EPR and Bell's theorems taken together.

In non-relativistic Quantum Mechanics, a more reasonable framework to assign a state vector to the single system is based on a stochastic modification of Schrödinger's equation [19]. Here, I will not discuss these kinds of interpretations since for the moment they have not been implemented to take into account Special Relativity in a completely consistent way. Moreover, they go somewhat beyond the purposes of the present paper since they suppose the introduction of new physics beyond QFT.

II. The remaining possibility for interpreting QFT without introducing new physics is to assume that the state vector does not describe the single system (which I will also call event), but only describes an ensemble of identically prepared copies of the system (more precisely, statistical operators should be used, since photons uncertainty prevents the preparation of a pure state as discussed below). This minimal statistical interpretation is clearly more economical than orthodox ones that use the unnecessary assumption that the state vector also describes the single systems. Moreover, it is very natural in QFT: for instance, if we consider a single particle that can decay, the theory is not able to say when it will actually decay, which direction the particles 
that are produced in the decay process will take, and which of the possible decay channels will be chosen. ${ }^{9}$ Similarly, in a Young double slit experiment, we are not able to predict anything about the single particle that will hit the screen in a random position.

As a matter of fact, the photons uncertainty itself implies that QFT does not make any prediction on the single event (i.e. on the single copy of the system), with the exception of the charges and masses of the particles that will possibly appear. However, QFT predicts probability rates and cross sections that can be compared with the frequencies of the results for the repetition of an experiment on a statistical ensemble of equally-prepared copies of the considered system. Therefore, it is natural (or at least conservative) to assume that the state vector only describes the ensemble, and not the single copy of the system (that we do not describe at all).

Of course, very convincing arguments for a statistical interpretations of the quantum theory have already been provided in literature, see e.g. refs. [8, 20, 21] and references therein. The most important is the fact that it naturally removes the measurement problem. Let me briefly summarize how this actually occurs.

For simplicity's sake, I will consider the measurement of an observable $\mathcal{A}$ in a two dimensional space of states. Let $\alpha_{1}$ and $\alpha_{2}$ be the (different) eigenvalues of $\mathcal{A}$, and let $\left|\alpha_{1}\right\rangle$, $\left|\alpha_{2}\right\rangle$ the corresponding eigenstates. If the (normalized) state of the object system is $|\psi\rangle=$ $c_{1}\left|\alpha_{1}\right\rangle+c_{2}\left|\alpha_{2}\right\rangle$, then the measurement of $\mathcal{A}$ will give either the result $\alpha_{1}$ with probability $\left|c_{1}\right|^{2}$ or the result $\alpha_{2}$ with probability $\left|c_{2}\right|^{2}$. After the measurement, the usual collapse postulate implies that the state is reduced to one of the eigenvectors of $\mathcal{A}$, depending on the result of the measurement: for instance, if the result is $\alpha_{1}$, the state after the measurement is reduced to $\left|\alpha_{1}\right\rangle$. The problem is that this postulate is incompatible with the Schrödinger equation (or the field equations) that imply a smooth and lineal evolution. In fact, let $|\Phi\rangle$ be the intial state of the measuring apparatus (that belongs to a different Hilbert space than that of the object system), and let $\Phi_{i}$ be its states when the results $\alpha_{i}$ are obtained, for $i=1,2$ respectively (let these vectors also describe the so-called "environment"). This means that if the initial state of the object system was one of the eigenvectors of $\mathcal{A}$, say $\left|\alpha_{1}\right\rangle$, then the corresponding initial state for the composite system made of our object + measurement device would be the tensor product $\left|\alpha_{1}\right\rangle|\Phi\rangle$, and the state after the measurement would be the tensor product $\left|\alpha_{1}\right\rangle\left|\Phi_{1}\right\rangle$. Similarly, $\left|\alpha_{2}\right\rangle|\Phi\rangle \rightarrow\left|\alpha_{2}\right\rangle\left|\Phi_{2}\right\rangle$ as a consequence of the measurement. If the measurement process could be described by the same linear evolution that applies to any physical process (the Schrödinger equation or the field equations), when the object system is not in an eigenstate of $\mathcal{A}$ the previous equations necessarily imply the following result for the measurement

$$
\left(c_{1}\left|\alpha_{1}\right\rangle+c_{2}\left|\alpha_{2}\right\rangle\right)|\Phi\rangle \rightarrow c_{1}\left|\alpha_{1}\right\rangle\left|\Phi_{1}\right\rangle+c_{2}\left|\alpha_{2}\right\rangle\left|\Phi_{2}\right\rangle .
$$

\footnotetext{
${ }^{9}$ Note that the result summarized in the previous section imply that there are always an infinite number of possible decay channels, involving an arbitrary number of additional photons; of course, usually there are also different channels involving other particles, such as neutrinos or different charged particles (for sufficient mass of the decaying particle).
} 
Now, if we assign a state to the individual system, as done in the usual "orthodox" interpretation of QM, we get a wrong result, since after measurement the system will be put in one of the two eigenvectors, not in the linear combination of eq. (5.1) (a measurement gives a definite result, not a superposition). This is the so-called measurement problem. The usual "orthodox" interpretation introduces the collapse of the state vector by hand, and therefore requires a nonlinear evolution law for the measurement process, which is different from the linear evolution that describes any other process (in other words, within this interpretation the measurement process cannot be described by the quantum theory itself). In my opinion, any interpretation that does not give a better answer to the measurement problem should simply be discarded, for several good reasons: we know from half a century of detector building that the particles are detected due to the usual electroweak and strong interactions, that are described by the linear equations of the Standard Model. Moreover, the collapse postulate is ultimately responsible for the paradoxes (that we might better call inconsistencies) of the quantum theory [8, 6]. In particular, it introduces a privileged reference frame, the Laboratory; the global description of the collapse is responsible for the supposed, paradoxical nonlocality of the theory, thus violating Special Relativity.

How does the statistical interpretation avoid the "measurement problem"? It assumes that the state vector only describes a statistical ensemble $\mathcal{E}$ of identical copies of the considered system, and it does not describe the single copy. The state after the measurement is the linear superposition of eq. (5.1). In fact, this state correctly describes the probabilities $\left|c_{1}\right|^{2}$ and $\left|c_{2}\right|^{2}$ for having obtained $\alpha_{1}$ or $\alpha_{2}$. Moreover, the single event has a definite result, although the theory does not predict it. The events that have given the result $\alpha_{1}$ form a subensemble $\mathcal{E}_{1}$ of $\mathcal{E}$, while those that gave $\alpha_{2}$ correspond to a different subensemble $\mathcal{E}_{2}$ (with $\mathcal{E}=\mathcal{E}_{1} U \mathcal{E}_{2}$ ). After the measurement, the experimenter (or the measuring device) selects only the events that have given a particular result, say $\alpha_{1}$, and this corresponds to reducing the statistical ensemble to $\mathcal{E}_{1}$. After this selection of the events, the state vector is the one that describes the new ensemble $\mathcal{E}_{1}$, which is $\left|\alpha_{1}\right\rangle\left|\Phi_{1}\right\rangle$, as in the usual collapse postulate (but here we have no paradox since we are not associating such a state to the individual system and we did not need a nonlinear evolution during the measurement process).

I recommend the excellent reviews [8, 20, 21] for a more complete discussion in terms of mixed states (which may be more fundamental than the pure states). Note that in ref. [8] the EPR paradox led to the conclusion that the quantum theory was incomplete and had to be completed: the single system had to have precise values of anticommuting observables like momenta and positions. Here, we do not need this assumption. QFT satisfies the EPR condition of completeness, without introducing additional variables. It is a strange kind of completeness: it allows for less reality than ordinary QM. Thus QFT can only be used to predict probabilities, statistical averages, correlations (that can be computed as usual through Feynman diagrams). Although I prefer to use the term "statistical", this is essentially the "correlation interpretation" described in ref. [6] (see also ref. [22]).

Incidentally, within this interpretation the world (i.e. the single event) has an objective consistency, independently of the possible measurements (no need to rely on any mysterious "quantum consciousness"). We merely cannot describe it but statistically. 
As a result, the measurement process can be considered a physical process involving quantum systems, that can be described by the quantum theory itself. On the other hand, the viability of such a description has been confirmed explicitly by recent studies, relying for the moment on the nonrelativistic QM (see e.g. [23] and references therein). Here, I will generalize this conclusion by assuming that the measurement process may be described precisely by the Standard Model itself, in agreement with the actual experience of Particle Physicists. This implies that QFT with this statistical interpretation is a fully local theory. In fact, causality and locality are satisfied by the Green functions of the theory (and then by the scattering matrix) [1, 2]. In other words, there is no possible source of nonlocality in the theory. You get back what you put in: if you put the nonlocal collapse, you get nonlocality. When we only use the local QFT interactions even to describe the measuring process, we get a fully local theory.

Note that in ref. [8] the EPR theorem led to the conclusion that the quantum theory had to be completed: the single system had to have precise values of anticommuting observables like momenta and positions, which played the role of hidden variables. As I have commented in the introduction, this was precisely the kind of solution that Einstein tended to favor, however (at least in its radical version) it is ruled out by Bell's theorem. Here, after removing EPR theorem, we do not need to introduce hidden variables. Ironically, we have come to a similar conclusion as EPR (the state vector does not describe the single system), although now we do not need to provide a more complete description! This fact may have important philosophical consequences.

It has been claimed that in statistical interpretations which do not introduce hidden variables "all the systems that have the same wave function are identical, since nothing differentiates them" [24]. Such a criticism would be correct if we did attribute the wave function to the single system. In a purely probabilistic theory, the only conclusion that could be reached with this kind of consideration would be that the minimal statistical interpretation does not explain why the measurements give sharp, different values, although it allows for this. On the other hand, the unique known kind of interpretations that would give a real "explanation" would be those based on hidden variables, which have problems with Bell's theorem (an exception is Bohm's theory, which I will not consider here since it is non-relativistic from the beginning and it has not been implemented to an interpretation of QFT). May be that we will never solve this mystery, which might reach the limits of science, although I hope that some progress will be provided with a possible Theory of Everything. For the moment, QFT with its statistical interpretation is not an explanation, but at least it is a consistent (and local) description.

\section{The EPR correlations in QFT}

Although we have already seen that QFT with the statistical interpretation is a local theory, it is interesting to discuss how this fact is shown in the EPR correlations that it predicts, whose supposed nonlocality is thought to be proven by the generalized Bell's Theorem (the only remaining argument after the removal of the EPR+Bell argument). 
Let us assume for simplicity that in an EPR-Bohm experiment the data analysis only records the "coincident events" with a particle A appearing in detector $O_{A}$ and a (say different) particle $\mathrm{B}$ appearing in $O_{B}$ (but all the following discussion can be easily generalized to the case of also considering the coincident events when $\mathrm{B}$ is caught by $O_{A}$ and $\mathrm{A}$ is caught by $O_{B}$, and to the case of $\mathrm{A}$ and $\mathrm{B}$ being identical, indistinguishable particles). I will call $\mathcal{E}$ the statistical ensemble of such coincident events, that have to be selected by two local measurements on both particles A and B. Suppose that $O_{A}$ measures the component $\vec{S}(A) \cdot \vec{a}$ of the spin of $\mathrm{A}$, and $O_{B}$ measures the component $\vec{S}(B) \cdot \vec{b}$ of the spin of B. Let $s_{a}= \pm \hbar / 2$ and $s_{b}= \pm \hbar / 2$ indicate the corresponding eigenvalues (i.e. the possible results of a measurement) of these spin projections. ${ }^{10}$

Now, the statistics over the ensemble $\mathcal{E}$ allows for the experimental evaluation of the spin correlations $\langle\vec{S}(A) \cdot \vec{a} \vec{S}(B) \cdot \vec{b}\rangle$. In QFT, such a correlation function has to be computed from the rate $\Gamma\left(s_{a}, s_{b}\right)$ for those coincident events where particle A and B are found with definite values $s_{a}$ and $s_{b}$ of their spin components. To give an example, I will assume that $\mathrm{A}$ and $\mathrm{B}$ are created in the decay process of a particle $\mathrm{X}$ (e.g. we may consider the decay of the possible Higgs boson into an electron-positron pair, that can be calculated in a straightforward way in the Standard Model), but the whole discussion which follows can be easily generalized e.g. to the case when A and B arise from a scattering process. I will compute the correlation at the lowest order, neglecting the rates for the creation of additional photons in coincidence with $\mathrm{A}$ and $\mathrm{B}$. In fact, although the effect of these additional photons was important for removing the EPR incompleteness argument, the contribution of the diagrams involving additional photons to the spin correlations is small [11], due to the fact that their rates are suppressed by powers of the fine structure constant and by a phase space factor depending on the small solid angles $\Omega_{A}$ and $\Omega_{B}$ intercepted by the detectors as seen from the production point (as far as we consider only the ensemble $\mathcal{E}$ ). A good approximation for the relevant QFT rate will be

$$
\Gamma\left(s_{a}, s_{b}\right) \simeq \frac{c^{2}}{2(2 \pi)^{2} \hbar M_{X}} \int_{\Omega_{A}} \frac{d^{3} \vec{p}_{A}}{2 E_{A}} \int_{\Omega_{B}} \frac{d^{3} \vec{p}_{B}}{2 E_{B}}\left|\mathcal{M}\left(\vec{p}_{A}, s_{a}, \vec{p}_{B}, s_{b}\right)\right|^{2} \delta^{4}\left(p_{A}+p_{B}-p_{X}\right)
$$

where the variables of integration $p_{A}=\left(E_{A} / c, \vec{p}_{A}\right), p_{B}=\left(E_{B} / c, \vec{p}_{B}\right)$ correspond to the four momenta of the two particles $\mathrm{A}$ and $\mathrm{B}$, and $M_{X}$ is the mass of the decaying particle that produces them (see e.g. eq. (A.57) of ref. [25]). On the other hand, $\mathcal{M}\left(\vec{p}_{A}, s_{a}, \vec{p}_{B}, s_{b}\right)$ is the Feynman amplitude for the process. Assuming that the two detectors are placed in exactly opposite directions and intercept similar solid angles, we can perform the integrals and get

$$
\Gamma\left(s_{a}, s_{b}\right) \simeq \frac{\Omega_{A}}{4 \pi} \frac{\left|\vec{p}_{A}\right|}{8 \pi \hbar c M_{X}^{2}}\left|\mathcal{M}\left(\vec{p}_{A}, s_{a},-\vec{p}_{A}, s_{b}\right)\right|^{2}
$$

where now

$$
\left|\vec{p}_{A}\right|=c M_{X}^{-1} \sqrt{M_{X}^{4}+M_{A}^{4}+M_{B}^{4}-2 M_{X}^{2} M_{A}^{2}-2 M_{X}^{2} M_{B}^{2}-2 M_{A}^{2} M_{B}^{2}}
$$

is the modulus of the momentum, as given by energy conservation (here we have chosen the rest frame of the decaying particle).

\footnotetext{
${ }^{10}$ More precisely, we understand that a basis of Dirac spinors has to be used.
} 
Restricting ourselves to the statistical ensemble $\mathcal{E}$, we can define the probability of obtaining $s_{a}$ and $s_{b}$ by dividing the rate in eq. (6.2) by the total rate corresponding to this statistical ensemble, i.e.

$$
\mathcal{P}\left(s_{a}, s_{b}\right) \equiv \frac{\Gamma\left(s_{a}, s_{b}\right)}{\sum_{s_{a}^{\prime}= \pm \hbar / 2} \sum_{s_{b}^{\prime}= \pm \hbar / 2} \Gamma\left(s_{a}^{\prime}, s_{b}^{\prime}\right)} \simeq \frac{\left|\mathcal{M}\left(\vec{p}_{A}, s_{a},-\vec{p}_{A}, s_{b}\right)\right|^{2}}{\sum_{s_{a}^{\prime}= \pm \hbar / 2} \sum_{s_{b}^{\prime}= \pm \hbar / 2}\left|\mathcal{M}\left(\vec{p}_{A}, s_{a}^{\prime},-\vec{p}_{A}, s_{b}^{\prime}\right)\right|^{2}}
$$

Now, in QFT the Feynman amplitude $\mathcal{M}$ is invariant under the full Lorentz group (transforming the momenta and spin/helicities as shown in ref. [1]). In particular, it is invariant under rotations, thus it conserves angular momentum. In the usual EPR-Bohm experiment we assume that the decaying system $M_{X}$ has zero angular momentum, thus $\mathrm{A}$ and $\mathrm{B}$ are created in a spin-singlet state (remember that we are neglecting any possible additional particles in this computation). Therefore, independently of the explicit form of the amplitude $\mathcal{M}$, that depends on the particular process under consideration, we get

$$
\mathcal{P}\left(+_{a},+_{b}\right)=\mathcal{P}\left(-{ }_{a},-{ }_{b}\right) \simeq \frac{1}{2} \sin ^{2}\left(\frac{\theta}{2}\right), \quad \mathcal{P}\left(+_{a},-{ }_{b}\right)=\mathcal{P}\left(-{ }_{a},+_{b}\right) \simeq \frac{1}{2} \cos ^{2}\left(\frac{\theta}{2}\right),
$$

where $\theta$ is the angle between the orientations $\vec{a}$ and $\vec{b}$ of the spin-measuring devices, and I have indicated $\pm \hbar / 2$ with the sign \pm . We can now evaluate the spin-spin correlation (for the ensemble $\mathcal{E}$ ) as

$$
\left\langle s_{a} s_{b}\right\rangle=\sum_{s_{a}= \pm \hbar / 2} \sum_{s_{b}= \pm \hbar / 2} \mathcal{P}\left(s_{a}, s_{b}\right) s_{a} s_{b} \simeq-\frac{\hbar^{2}}{4} \cos \theta .
$$

This is the same result obtained in the usual QM treatment [21, [0]. There are however two important differences. Firstly, now we know that it needs two local measurements on both $\mathrm{A}$ and $\mathrm{B}$, and that it is merely an approximation. Secondly, we see that this result arises from eq. (6.3), which only depends on the invariant Feynman amplitude $\mathcal{M}$. Now, in the computation of the Feynman amplitudes all the conservation laws have a local origin [1, 2], and in particular the global angular momentum conservation is a consequence of the local conservation, as we expected from the discussion of the previous section.

This is also reflected by the fact that the correlation does not depend on the distance: it is the same for large and small distances. It is true that one can then change the axis $\vec{a}$ and $\vec{b}$ of the distant detectors, but the correlation that is computed with the new axis could also be computed for small distances. The correlation has then to be created in the production process; although it depends nontrivially on the experimental arrangement in $O_{A}$ and $O_{B}$, it does not corresponded to any influence at a distance.

In fact, eq. (6.4) satisfies the only real locality conditions that are imposed by Special Relativity, according to Ballentine and Jarrett [18]:

$$
\sum_{s_{b}} \mathcal{P}\left(s_{a}, s_{b}\right)=\frac{1}{2}, \quad \text { for } \quad s_{a}= \pm \frac{\hbar}{2} ; \quad \sum_{s_{a}} \mathcal{P}\left(s_{a}, s_{b}\right)=\frac{1}{2}, \quad \text { for } \quad s_{b}= \pm \frac{\hbar}{2},
$$

valid for whatever choice of orientations $\vec{a}$ and $\vec{b}$ of the detectors. In particular, detector $O_{A}$ observes the same distribution of probability independently of the settings of the distant detector $O_{B}$. This is also sufficient to prevent the possibility of any superluminal 
communication. On the other hand, eq. (6.5) coincides with the usual QM result, that agrees with the actual experiments and violates Bell's inequalities. As Ballentine and Jarrett have argued, this means that it is not possible to find a set of parameters $\lambda$, carrying the information on the state and the initial conditions, so that the probability can be factorized as

$$
\mathcal{P}\left(s_{a}, s_{b}, \lambda\right)=\sum_{s_{b}^{\prime}} \mathcal{P}\left(s_{a}, s_{b}^{\prime}, \lambda\right) \sum_{s_{a}^{\prime}} \mathcal{P}\left(s_{a}^{\prime}, s_{b}, \lambda\right),
$$

where the dependence on $\lambda$ in the probabilities corresponds to partitioning the ensemble of the events in subensembles that have equal conditions $\lambda$. The impossibility of finding such a decomposition implies that the measurement on A gives additional information about the measurement on $\mathrm{B}$, with respect to the information that can be contained in the state preparation. Ballantine and Jarrett, implicitly using a statistical interpretation, have proved that this fact should not be interpreted as a sign of nonlocality, but that it only proves that the quantum theory is less predictive than it would be using the state vector to describe the single system. This reduction of predictivity is unavoidable as far as we limit the description only to the statistics on the ensemble of copies of the system.

A complete discussion can be found in ref. [18]. Here, I will merely point out that the measurement on A unavoidably gives an information for the measurement on $\mathrm{B}$, and this information depends on the orientation $\vec{a}$ of the apparatus $O_{A}$. In fact, let me call $\mathcal{E}\left(s_{a}, s_{b}\right)$ the partition of the total ensemble $\mathcal{E}$ of the coincident events, where for instance $\mathcal{E}\left(+_{a},+_{b}\right)$ is the ensemble of the events that have given the results $s_{a}=+\hbar / 2, s_{b}=$ $+\hbar / 2$ after the measurement. This partition depends on the choice of the orientations $\vec{a}$ and $\vec{b}$ of the apparatuses, and changes if we modify one of these orientations. Since the results of the single measurements are not predicted by the theory, a partition can be obtained only after actually performing the measurements. The probabilities $\mathcal{P}\left(s_{a}, s_{b}\right)$ can be computed by counting the number of events that belong to the different subensembles $\mathcal{E}\left(s_{a}, s_{b}\right)$ of the partition of $\mathcal{E}$ and dividing by the total number of events in $\mathcal{E}$ (of course, this computation would give the correct probabilities only in the limit where $\mathcal{E}$ counts infinite events). It is clear that these probabilities depend on the partition that is chosen, in other words they depend nontrivially (and in a "nonseparable" way) on the orientations of the two apparatuses and on the results of the single measurements, without implying any nonlocality. ${ }^{11}$ On the other hand, although it cannot be generalized to prove nonlocality, Bell's Theorem may remain an important argument against local determinism [18].

\section{Conclusions}

In a previous paper, I have shown that the Standard Model of Particle Physics prevents the production of states having a definite number of particles, contradicting a basic assumption of the EPR argument. Here, this result has been used to remove one of the supposed proofs

\footnotetext{
${ }^{11}$ In Ballentine and Jarrett's paper [18], the final considerations on the EPR incompleteness argument should be updated. Now, QFT satisfies the EPR criterion of completeness, although it allows for less reality than a classically complete theory. On the other hand, QFT does not predict anything on the single event, and it is "predictively incomplete" 18 .
} 
of nonlocality as well, that based on the EPR argument and on the original Bell's Theorem. The great uncertainty of QFT also provides a hint for a (minimal) statistical interpretation that renounces describing the single events. Such an interpretation does not assume any global collapse of the state vector and solves the "measurement problem" in a natural way, since it allows for describing the measurement as a physical quantum process, in agreement with recent results [23] and with the experience of particle physicists. This also allows for a complete recovery of locality and causality without introducing hidden variables. I have then shown how the EPR correlations should be computed in QFT using Lorentz invariant Feynman amplitudes, and recalled the argument by Ballentine and Jarrett against the wild generalizations of Bell's Theorem. The latter theorem may still be used in its original formulation to rule out local determinism, but it cannot prevent the probabilistic QFT to be a local theory. We have no guarantee that a better understanding of the quantum correlations will ever be found, although it can be hoped that this will eventually be achieved in a possible ultimate Theory of Everything. Further research in this direction will be most welcome (e.g. following the ideas given in refs. [26, 27]). In any case, I think that locality and Lorentz symmetry will not be so badly violated as to allow for whatever instantaneous effect between very distant objects, since QFT will presumably remain a good approximation in the "low energy" regime (corresponding precisely to the long distance behavior). On the other hand, these results can be expected to be maintained or even strengthened in a possible quantum gravity, since General Relativity has been argued to require a local interpretation of the correlations [28].

\section{Acknowledgments}

I thank Humberto Michinel for very useful and stimulating discussions, and Rebecca Ramanathan and Ruth García Fernández for help.

\section{References}

[1] S. Weinberg, The quantum theory of fields, vol. I Cambridge University Press, 1995.

[2] S. Weinberg, The quantum theory of fields, vol. II Cambridge University Press, 1996.

[3] Y. Aharonov and D.Z. Albert, Can we make sense out of the measurement process in relativistic quantum mechanics?, Phys. Rev. D 24 (1981) 359;

Y. Aharonov, D.Z. Albert and L. Vaidman, Measurement process in relativistic quantum theory, Phys. Rev. D 34 (1986) 1805.

[4] H.P. Stapp, Nonlocal character of quantum theory, Am. J. Phys. 65 (1997) 300.

[5] A. Zeilinger, Experiment and the foundations of quantum physics, Rev. Mod. Phys. 71 (1999) S288.

[6] F. Laloë, Do we really understand quantum mechanics? Strange correlations, paradoxes, and theorems, Am. J. Phys. 69 (2001) 655.

[7] V. Scarani, W. Tittel, H. Zbinden and N. Gisin, The speed of quantum information and the preferred frame: analysis of experimental data, Phys. Lett. A 276 (2000) 1. 
[8] L.E. Ballentine, The statistical interpretation of quantum mechanics, Rev. Mod. Phys. 42 (1970) 358.

[9] A. Einstein, B. Podolsky and N. Rosen, Can quantum mechanical description of physical reality be considered complete?, Phys. Rev. 47 (1935) 777.

[10] D. Bohm, Quantum theory Prentice-Hall, 1951.

[11] D. Tommasini, Photons uncertainty removes Einstein-Podolsky-Rosen paradox, quant-ph/0202175.

[12] A. Einstein, in Albert Einstein, philosopher-scientist, P.A. Schilpp ed., Library of Living Philosophers, Evanston, 1949.

[13] A. Peres, Quantum theory: concepts and methods, Kluver Academic Publishers, 1995.

[14] J. Bell, On the Einstein-Podolsky-Rosen paradox, Physics 1 (1964) 195.

[15] J. Bell, On the problem of hidden variables in quantum mechanics, Rev. Mod. Phys. 38 (1966) 447 .

[16] A. Aspect, J. Dalibard and G. Roger, Experimental test of Bell's inequalities using time varying analyzers, Phys. Rev. Lett. 49 (1982) 1804.

[17] D. Bohm, A suggested interpretation of the quantum theory in terms of hidden variables 1, Phys. Rev. 85 (1952) 166.

[18] L.E. Ballentine and J.P. Jarrett, Bell's theorem: does quantum mechanics contradict relativity?, Am. J. Phys. 55 (1987) 696.

[19] G.C. Ghirardi, A. Rimini and T. Weber, A unified dynamics for micro and macro systems, Phys. Rev. D 34 (1986) 470.

[20] F.J. Belinfante, Measurements and time reversal in objective quantum theory, Pergamon, 1975.

[21] B.H. Bransden and C.J. Joachain, Quantum mechanics, 2nd edition Prentice Hall, 2000.

[22] C.A. Fuchs and A. Peres, Quantum theory needs no 'interpretation', Physics Today 53 (2000) 70 .

[23] C. Presilla, R. Onofrio and U. Tambini, Measurement quantum mechanics and experiments on quantum zeno effect, Ann. Phys. (NY) 248 (1996) 95 cond-mat/9603182.

[24] B. d'Espagnat, Veiled reality Addison-Wesley, 1995.

[25] M.E. Peskin and D.V. Schroeder, An introduction to quantum field theory Perseus Books, 1995.

[26] R. Gambini and R.A. Porto, Relational reality in relativistic quantum mechanics, Phys. Lett. A 294 (2002) 129 quant-ph/0105146.

[27] M. Czachor and H. D. Doebner, Correlation experiments in nonlinear quantum mechanics, quant-ph/0110008.

[28] H. von Borzeszkowski and M.B. Mensky, EPR effect in gravitational field: nature of nonlocality, Phys. Lett. A 269 (2000) 204. 